\title{
Complications are reduced with a protocol to standardize timing of fixation based on response to resuscitation
}

Heather A. Vallier ${ }^{1 *}$, Timothy A. Moore ${ }^{1,2}$, John J. Como ${ }^{3}$, Patricia A. Wilczewski ${ }^{3}$, Michael P. Steinmetz ${ }^{4}$ Karl G. Wagner ${ }^{5}$, Charles E. Smith ${ }^{5}$, Xiao-Feng Wang ${ }^{1}$ and Andrea J. Dolenc ${ }^{1}$

\begin{abstract}
Background: Our group developed a protocol, entitled Early Appropriate Care (EAC), to determine timing of definitive fracture fixation based on presence and severity of metabolic acidosis. We hypothesized that utilization of EAC would result in fewer complications than a historical cohort and that EAC patients with definitive fixation within $36 \mathrm{~h}$ would have fewer complications than those treated at a later time.

Methods: Three hundred thirty-five patients with mean age 39.2 years and mean Injury Severity Score (ISS) 26.9 and 380 fractures of the femur $(n=173)$, pelvic ring $(n=71)$, acetabulum $(n=57)$, and/or spine $(n=79)$ were prospectively evaluated. The EAC protocol recommended definitive fixation within $36 \mathrm{~h}$ if lactate $<4.0 \mathrm{mmol} / \mathrm{L}, \mathrm{pH} \geq 7.25$, or base excess (BE) $\geq-5.5 \mathrm{mmol} / \mathrm{L}$. Complications including infections, sepsis, DVT, organ failure, pneumonia, acute respiratory distress syndrome (ARDS), and pulmonary embolism (PE) were identified and compared for early and delayed patients and with a historical cohort.

Results: All 335 patients achieved the desired level of resuscitation within 36 h of injury. Two hundred sixty-nine (80 \%) were treated within $36 \mathrm{~h}$, and 66 had protocol violations, treated on a delayed basis, due to surgeon choice in $71 \%$. Complications occurred in $16.3 \%$ of patients fixed within $36 \mathrm{~h}$ and in $33.3 \%$ of delayed patients $(p=0.0009)$. Hospital and ICU stays were shorter in the early group: 9.5 versus 17.3 days and 4.4 versus 11.6 days, respectively, both $p<0.0001$. This group of patients when compared with a historical cohort of 1443 similar patients with 1745 fractures had fewer complications ( 16.3 versus $22.1 \%, p=0.017$ ) and shorter length of stay (LOS) $(p=0.018)$.

Conclusions: Our EAC protocol recommends definitive fixation within $36 \mathrm{~h}$ in resuscitated patients. Early fixation was associated with fewer complications and shorter LOS. The EAC recommendations are safe and effective for the majority of severely injured patients with mechanically unstable femur, pelvis, acetabular, or spine fractures requiring fixation.
\end{abstract}

Keywords: Polytrauma, Fixation timing, Damage control, Femur fracture, Pelvis fracture, Spine fracture, Resuscitation

\section{Background}

Early stabilization of femur, pelvis, acetabulum, and spine fractures minimizes pulmonary and other complications, while damage control tactics may provide provisional stability in patients too unstable to tolerate definitive surgery [1-18]. Currently, controversy exists regarding indications for damage control procedures

\footnotetext{
* Correspondence: hvallier@metrohealth.org

'Department of Orthopaedic Surgery, MetroHealth Medical Center affiliated with Case Western Reserve University, 2500 MetroHealth Drive, Cleveland, $\mathrm{OH}$ 44109, USA

Full list of author information is available at the end of the article
}

$[11-13,19,20]$. Unnecessary delay of definitive care has been associated with pulmonary complications, deep venous thrombosis, skin breakdown, and sepsis [2, 4-6, 18, 21-23]. Delay is also associated with longer hospital stays and ventilation times $[1,2,10,14,17,18,24]$. Ultimately, delay can generate increased costs of care and opportunity costs in terms of limited access for other patients to a given hospital system [25].

We developed a protocol to determine timing of definitive fracture care based on the presence and severity of metabolic acidosis as measured by arterial $\mathrm{pH}$ or base excess and/or venous lactate [26]. Key features of this 
protocol are simplicity, ease of assessment, universal application to all patients, and agreement among all trauma providers in the implementation thereof. The purpose of this project was to prospectively assess the safety and feasibility of this protocol, defined as Early Appropriate Care (EAC). We hypothesized that (1) utilization of EAC would be associated with fewer complications than a historical cohort of similar patients and that (2) EAC patients treated definitively for their fractures of interest within $36 \mathrm{~h}$ of injury would have fewer complications than those who had protocol violations and treated on a delayed basis.

\section{Methods}

Three hundred thirty-five consecutive, skeletally mature patients with Injury Severity Score (ISS) $\geq 16$ and 380 fractures of the proximal or diaphyseal femur $(n=173)$, pelvic ring $(n=71)$, acetabulum $(n=57)$, and/or spine $(n=79)$ were treated surgically over 30 months. Associated injuries of the chest $(n=209)$, abdomen $(n=$ $97)$, and head $(n=192)$ were present. When the Abbreviated Injury Score (AIS) for a region was $\leq 2$, the injuries were defined as minor, while AIS $>2$, were considered severe. Fracture characteristics, associated injuries, medical co-morbidities, and the timing and techniques of provisional treatment and surgical procedures were documented.

\section{EAC protocol}

Inclusion criteria were patients with mechanically unstable fractures of the proximal or diaphyseal femur, pelvic ring, acetabulum, and/or spine requiring fixation. All patients had a presenting ISS $\geq 16$ and at least one of the following: injury to one or more other body systems, hemodynamic instability on presentation, defined by hypotension (systolic blood pressure <90), tachycardia (heart rate greater than 110), and/or immediate transfusion requirements. Exclusion criteria included fractures sustained from a low energy mechanism or secondary to neoplasm and those in skeletally immature patients.

The EAC protocol recommended definitive fixation of the fractures of interest within $36 \mathrm{~h}$ of injury, as long as initial acidosis had improved to at least one of the following: lactate $<4.0 \mathrm{mmol} / \mathrm{L}, \mathrm{pH} \geq 7.25$, or base excess (BE) $\geq-5.5 \mathrm{mmol} / \mathrm{L}$. The protocol parameters were developed via a model previously reported [26]. All patients were also required to be responding to resuscitation without pressor support. Venous lactate is routinely obtained at the time of presentation to our facility. Arterial blood gas measurements were obtained at the discretion of the treating general trauma surgeon. Labs were repeated a minimum of every $8 \mathrm{~h}$ until normal, both preoperatively and postoperatively.
In cases of persistent acidosis, damage control was recommended if fractures were amenable to this. Ongoing clinical and laboratory reassessment was performed, and a safe time for definitive fixation was determined by the attending trauma surgeon. In the event of ongoing active bleeding secondary to fracture, with failure to respond to conventional methods including splinting, transfusion, and angiography, the general trauma surgeon could recommend fixation or amputation as a life saving measure. For patients with two or more of the fractures of interest, the orthopedic trauma surgeon would propose a plan for sequence of fixation. The general trauma surgeon would review this plan and propose incorporation of any other procedures warranted prior to fracture care or within the same surgical setting. The trauma surgeon, in conjunction with the orthopedic trauma and anesthesia teams, would reassess the patient during the first of multiple procedures and sequentially thereafter to determine the safety of continuing surgery. Worsening acidosis would be considered an indication to delay additional procedures. Notably, some patients would have open extremity fractures for which a minimum of urgent debridement and irrigation followed by splinting and/or external fixation was anticipated in the initial surgical setting. Standard inpatient protocols for antibiotic usage, DVT prophylaxis, and nutrition were in place and unchanged throughout the study period.

\section{Measurements of outcome}

Complications included infections, sepsis, DVT, organ failure, and pulmonary complications: pneumonia, acute respiratory distress syndrome (ARDS), and pulmonary embolism (PE). Sepsis was defined by positive blood culture and at least two of the following: temperature > $38{ }^{\circ} \mathrm{C}$ or $<36{ }^{\circ} \mathrm{C}$, heart rate $>90$ beats/minute, respiratory rate $>20$ breaths/min, and white blood cell count $>12,000 /$ $\mathrm{mL},<4,000 / \mathrm{mL}$, or $>10 \%$ band forms [27]. Deep vein thrombosis proximal to the knee was diagnosed on duplex ultrasound, and PE was diagnosed on computed tomography. Acute renal failure was defined as a $50 \%$ increase in creatinine from baseline [28]. Multiple organ failure was defined as two or more organs in failure for a minimum of three consecutive days with a score of four or more points [29]. Acute respiratory distress syndrome was defined as $\mathrm{PaO}_{2} / \mathrm{FiO}_{2}$ ratio of less than 200 for more than four consecutive days with diffuse infiltrates on chest radiographs, in the absence of pneumonia [30]. Pneumonia was defined by quantitative culture obtained via bronchoscopy and bronchoalveolar lavage; decision to perform this procedure was made by the attending trauma intensivist and was generally prompted by new pulmonary infiltrate on plain chest radiograph in conjunction with purulent 
sputum, temperature greater than $38{ }^{\circ} \mathrm{C}$ and/or a white blood count greater than $10,000 / \mathrm{mL}$ [30].

An adjudication committee consisting of physicians from anesthesiology, orthopedic surgery, neurosurgery, and trauma surgery/surgical critical care independently reviewed all records at 6-month intervals. Adherence to the protocol was assessed, including reasons for delay of surgery. None of the adjudicating physicians participated in the collection or analysis of research data.

\section{Statistical analysis}

Continuous variables were compared using the two sample $t$ test, and categorical variables were compared using the Pearson's chi-square test. The study group was divided into two groups based upon timing of fixation: before or after $36 \mathrm{~h}$. For the binary outcome measures, logistic regression models were created. For length of stay (LOS) and ventilation times, generalized linear regression models with negative binominal distribution were used. This is due to the fact that days are nonnegative integers and have a right-skewed distribution. Both univariate and multivariate logistic regression models and negative-binomial regression models were applied to find potential risk factors. Risks factors included patient age, gender, ISS, type of fracture, and type and severity of other system injuries, including those to the head, chest, and abdomen. All analyses were performed by a statistician not involved in the treatment of the patients. The SAS statistical package was used (Version 9.3 for Unix., SAS Inc., Cary, NC, USA). The level of statistical significance was set at $p<0.05$ (two-tailed).

\section{Statement of ethical approval}

Institutional Review Board approval was obtained from the MetroHealth Institutional Review Board prior to initiating this study: \#IRB07-01157.

All devices used in this study are FDA-approved.

\section{Results}

Three hundred thirty-five consecutive patients with 380 fractures were prospectively assessed and treated according to EAC criteria. This included 239 men and 96 women with mean age of 39.2 years and mean ISS of 26.9 (Table 1) and injuries to other systems (Table 2). Two hundred sixty-nine patients $(80 \%)$ had 301 fractures treated within $36 \mathrm{~h}$ of injury, according to the EAC protocol, and 66 (20\%) patients had 79 fractures fixed on a delayed basis (protocol violations), although all patients met the desired EAC resuscitation parameters, with improvement of acidosis, within $36 \mathrm{~h}$ of injury. Femoral fractures were more likely to be treated within $36 \mathrm{~h}(90 \%, p=0.0003)$, and spinal fractures were more likely to be treated later than $36 \mathrm{~h}$ from injury (34\%, $p<0.0001)$. Patients with spine fractures which were treated more than $36 \mathrm{~h}$ after injury received more blood transfusions versus those treated earlier $(9.7$ versus $4.3 \mathrm{U}, p=0.032$ ); however, other fracture subtypes had no differences in the number of units of blood transfused when early and delayed groups were compared.

Reasons for treatment after $36 \mathrm{~h}$ following injury are shown in Table 3. Surgeon choice to delay was the most common reason, occurring in $71 \%$. An operating room was not available in four other cases (6.1\%). Five patients were determined to be medically unstable: two requiring pressor support for hypotension, two with concern for myocardial infarction, and one with severe hyperkalemia. Two patients were treated on a delayed basis due to traumatic brain injury with severely elevated intracranial pressures (3.0 \%).

Three patients $(0.9 \%)$ had protocol violations due to definitive fixation prior to meeting the resuscitative criteria. They were included with the patients treated definitively within $36 \mathrm{~h}$ in the final analysis. One other patient underwent damage control external fixation of a femur shaft fracture due to persistent acidosis and elevated intracranial pressure. Her systemic status never improved, and she died from her head injury 4 days later. She never received definitive fixation; thus, she was excluded from further analysis.

Complications occurred in $16.3 \%$ of patients treated within $36 \mathrm{~h}$ and in $33.3 \%$ of delayed patients $(p=$ $0.0009)$, including pneumonia in 8.2 and $13.6 \%(p=$ 0.09 , Table 4), respectively. Multivariate logistic regression accounted for potential confounders including age, gender, ISS, type of fracture, and type and severity of other system injuries, and patients with fixation within $36 \mathrm{~h}$ had a lower total complication rate $(p=0.0058)$. Greater age was also associated with a higher complication rate after multivariate analyses $(p=0.0074)$. The delayed group had more sepsis (18.2 versus $2.2 \%, p<$ 0.0001 ) when compared to patients treated within $36 \mathrm{~h}$. Delayed surgery was also associated with acute renal failure (7.6 versus $0.4 \%, p=0.0001$ ). Death occurred overall in $1.5 \%$ of patients at mean 21.2 days. Causes included: respiratory failure in two, sepsis in one, and organ failure in two. Two patients died after hospital discharge (5 and 7 months) of unknown causes. Deep venous thrombosis occurred in $1.8 \%$ of patients overall; all were patients in the early fixation group.

Sixty-two percent (209 of 335) of our patients had a chest injury; 88 ( $42.1 \%$ of 209$)$ were minor injuries and 121 (57.9\% of 209) were severe. Mean time from injury to fixation was no different when patients with and without chest injury were compared (29.2 versus 25.5 h; $p=0.53$ ). However, patients with chest injuries were significantly more likely to have pulmonary complications (17.2 \%, $p=0.001)$ and any complication 
Table 1 Demographic information on EAC patients treated with definitive fixation within $36 \mathrm{~h}$ of injury versus those treated later

\begin{tabular}{|c|c|c|c|c|}
\hline & $\begin{array}{l}\text { All patients: } 335 \text { with } \\
380 \text { fractures }\end{array}$ & $\begin{array}{l}\text { Definitive fixation } \leq 36 \text { h: } 269 \\
\text { patients with } 301 \text { fractures }\end{array}$ & $\begin{array}{l}\text { Definitive fixation }>36 \mathrm{~h}: 66 \\
\text { patients with } 79 \text { fractures }\end{array}$ & $p$ value \\
\hline Male & $239(71 \%)$ & $199(74 \%)$ & $40(61 \%)$ & 0.04 \\
\hline Female & $96(29 \%)$ & $70(26 \%)$ & $26(39 \%)$ & \\
\hline Mean age (years) & 39.2 & 39.1 & 39.6 & 0.31 \\
\hline Range & 14 to 91 & 14 to 91 & 17 to 82 & \\
\hline Mean ISS & 26.9 & 25.1 & 34 & 0.0005 \\
\hline Range & 16 to 66 & 16 to 66 & 16 to 66 & \\
\hline \multicolumn{5}{|l|}{ Mechanism of injury } \\
\hline MVC & 170 (51 \%) & 131 (49 \%) & $39(59 \%)$ & \\
\hline MCC & $52(16 \%)$ & $47(17 \%)$ & $5(7.6 \%)$ & \\
\hline Fall from height & $63(19 \%)$ & $54(20 \%)$ & $9(14 \%)$ & \\
\hline Pedestrian vs MVC & $20(6.0 \%)$ & $14(5.2 \%)$ & $6(9.1 \%)$ & \\
\hline GSW & $15(4.5 \%)$ & $14(5.2 \%)$ & 1 (1.5\%) & \\
\hline Crush & $8(2.4 \%)$ & $4(1.5 \%)$ & $4(6.1 \%)$ & \\
\hline Other & $7(2.1 \%)$ & $5(1.9 \%)$ & $2(3.0 \%)$ & \\
\hline \multicolumn{5}{|l|}{ Fractures } \\
\hline Femur & $173(52 \%)$ & $155(58 \%)$ & $18(27 \%)$ & $p=0.0003$ \\
\hline Pelvis & $71(21 \%)$ & $48(18 \%)$ & $23(35 \%)$ & $p=0.07$ \\
\hline Acetabulum & $57(17 \%)$ & $46(17 \%)$ & $11(17 \%)$ & $p=0.39$ \\
\hline Spine & 79 (24 \%) & $52(19 \%)$ & $27(41 \%)$ & $p<0.0001$ \\
\hline Patients with 1 fracture & $291(87 \%)$ & $236(88 \%)$ & $55(83 \%)$ & $p=0.35$ \\
\hline Patients with 2 fractures & $40(12 \%)$ & $31(12 \%)$ & $9(14 \%)$ & \\
\hline Patients with 3 fractures & $3(0.9 \%)$ & 1 (0.4\%) & $2(3.0 \%)$ & \\
\hline
\end{tabular}

ISS Injury Severity Score, MVC motor vehicle collision, MCC motorcycle crash, GSW gunshot wound

Percentages of all patients in each column with a given gender, mechanism, and type of fracture are shown in parentheses

(25.8\%, $p=0.0005)$. Eighty-three percent of the pulmonary complications in chest-injured patients occurred in those with severe injuries.

A separate analysis was undertaken to evaluate patients without chest injury $(n=126)$. When fixation was within $36 \mathrm{~h}$ of injury, $15 \%$ had complications versus $28 \%$ with later fixation $(p=0.15)$. Shorter ventilation times and ICU and total hospital stays were noted with early fixation (all $p<0.007$ ). When patients with no chest injury or chest injury AIS $\leq 2(n=88)$ were combined, significantly fewer complications were noted with early fixation $(p=0.004)$, in addition to shorter hospital stays and ventilation times (all $p<0.001$ ).

Seventy-nine patients were treated surgically for spine fractures. Thirty-one had spinal cord injury (39\%), with equal distributions in the early and delayed groups.

Table 2 Associated injuries. The numbers of patients with associated abdominal, chest, and/or head injuries are listed

\begin{tabular}{|c|c|c|c|c|}
\hline & Fixation within $36 \mathrm{~h}(n=269)$ & Fixation after $36 \mathrm{~h}(n=66)$ & Totals $(n=335)$ & \\
\hline Abdominal injury & $66(25 \%)$ & $31(47 \%)$ & $97(29 \%)$ & $p=0.0002$ \\
\hline Minor (AIS $\leq 2)$ & $37(14 \%)$ & $12(18 \%)$ & 49 (15\%) & NS \\
\hline Severe (AIS > 2) & $29(11 \%)$ & $19(29 \%)$ & $48(14 \%)$ & $p<0.0001$ \\
\hline Chest injury & 167 (62 \%) & $42(64 \%)$ & $209(62 \%)$ & NS \\
\hline Minor (AIS $\leq 2)$ & $75(28 \%)$ & $13(20 \%)$ & $88(26 \%)$ & NS \\
\hline Severe (AIS > 2) & $92(34 \%)$ & $29(44 \%)$ & $121(36 \%)$ & $p=0.14$ \\
\hline Head injury & $149(55 \%)$ & $43(65 \%)$ & $192(57 \%)$ & $p=0.08$ \\
\hline (GCS 9-15) & 109 (41\%) & $26(39 \%)$ & $135(40 \%)$ & NS \\
\hline$(\mathrm{GCS} \leq 8)$ & $40(15 \%)$ & $17(26 \%)$ & $57(17 \%)$ & $p=0.018$ \\
\hline
\end{tabular}

$P$ values are listed for comparisons of the groups treated within $36 \mathrm{~h}$ of injury versus later AIS Abbreviated Injury Score, GCS Glasgow Coma Scale 
Table 3 Reasons for surgical delay in patients treated definitively more than $36 \mathrm{~h}$ after injury. Sixty-six patients had definitive surgery for 79 fractures on a delayed basis. EAC resuscitation criteria were met in all cases within $36 \mathrm{~h}$

\begin{tabular}{ll}
\hline Reason for delay & Number of patients (\%) \\
\hline Surgeon choice & $47(71 \%)$ \\
Intensivist choice & $6(9.1 \%)$ \\
Medically unstable & $5(7.6 \%)$ \\
Operating room unavailable & $4(6.1 \%)$ \\
Severe head injury & $2(3.0 \%)$ \\
Patient choice & $2(3.0 \%)$ \\
\hline
\end{tabular}

Chest injury occurred in $59 \%$ of the spine fracture patients. Despite an equal proportion of chest-injured patients, with similar injury severity, complications occurred more frequently in patients with spine fractures versus other fractures $(28 \%, p=0.01$, Table 5). Early definitive spine fixation did not increase complications; in contrast, the mean time to fixation of spine fractures in patients without complications was 26 versus $68 \mathrm{~h}$ in patients who developed complications $(p<0.0001)$. Surgeon choice was the reason for delayed fixation in $61 \%$ of cases.

Additional analysis was performed to review patients who were not treated for spine fractures and to compare them with the thoracolumbar spine patients (Table 5). Mean age was 39 years for both groups, and mean ISS was 27.0 for spine patients and 26.8 for the other patients. Complications occurred in $33 \%$ in both groups when fixation was undertaken more than $36 \mathrm{~h}$ after injury. Similar to spine patients, fixation performed within $36 \mathrm{~h}$ of injury was associated with fewer complications in patients treated for fractures of the pelvis, acetabulum, and/or femur (14 versus $33 \%, p=0.002$ ). Notably, patients treated for femoral shaft fractures incurred fewer complications than patients treated for other fracture types $(6.9 \%$ overall and $5.8 \%$ when treated within $36 \mathrm{~h}$, both $p<0.0001$ ).

This entire group of EAC patients was compared with a historical cohort of 1443 similar patients with 1745 fractures treated at the same hospital between 2000 and 2007 (Table 4). A propensity score matching approach was used to account for confounding variables, including age, gender, and associated injuries. Pulmonary complications were noted in $12.8 \%$ of the matched group versus $10.7 \%$ of EAC patients, $p=0.15$. Complications occurred in $19.7 \%$ of all EAC patients versus $22.1 \%$ in the historical group, $p=0.17$. When comparing the EAC patients treated within $36 \mathrm{~h}$ with the matched historical cohort, fewer total complications (16.3 versus $22.1 \%$, $p=0.017)$ were noted with EAC. In comparing the patients treated within $36 \mathrm{~h}$ with the EAC protocol versus our historical group, pulmonary complications were noted in 10.8 versus $17.8 \%, p=0.09$, and significantly shorter ICU $(p=0.048)$ and hospital stays $(p=0.018)$ were seen for EAC patients.

Mean LOS was 10.8 days, with mean of 6.01 ICU days (Table 6). Both were significantly shorter in patients treated definitively within $36 \mathrm{~h}$ of injury: 4.35 versus 11.6 days and 9.52 versus 17.3 days (both $p<0.0001$ ). Negative-binomial regression models were applied to account for potential confounders, and all differences remained significant. Time of mechanical ventilation was also significantly shorter in the early group.

\section{Discussion}

Early definitive stabilization of femoral shaft fractures and other major skeletal injuries was advocated by various surgeons over 30 years ago [2, 5, 21]. Higher incidences of pneumonia, ARDS, fat embolism, longer hospital stays, and higher costs were associated with delayed fixation, and benefits of early fixation were more profound in patients with multiple system trauma versus

Table 4 Complications in EAC patients treated within $36 \mathrm{~h}$ versus later. Comparison is made with a previously described cohort of patients with the same types of fractures managed at the same trauma center

\begin{tabular}{lllll}
\hline & All EAC patients $(n=335)$ & EAC treated $\leq 36 \mathrm{~h}(n=269)$ & EAC treated $>36 \mathrm{~h}(n=66)$ & Historical comparison group $(n=1443)$ \\
\hline Any complication & 66 patients $(19.7 \%)$ & 44 patients $(16.3 \%)$ & 22 patients $(33.3 \%)$ & 319 patients $(22.1 \%)$ \\
& with 90 complications & with 57 complications & with 32 complications* & with 354 complications \\
Pneumonia & $31(9.3 \%)$ & $22(8.2 \%)$ & $9(14 \%)$ & $118(8.2 \%)$ \\
DVT & $6(1.8 \%)$ & $6(2.2 \%)$ & 0 & $92(6.4 \%)$ \\
PE & $8(2.4 \%)$ & $7(2.6 \%)$ & $1(1.5 \%)$ & $22(1.5 \%)$ \\
ARDS & $5(1.5 \%)$ & $4(1.5 \%)$ & $1(1.5 \%)$ & $33(2.3 \%)$ \\
ARF & $6(1.8 \%)$ & $1(0.37 \%)$ & $5(7.6 \%)^{*}$ & $24(1.7 \%)$ \\
MOF & $2(0.60 \%)$ & $1(0.37 \%)$ & $1(1.5 \%)$ & $5(0.35 \%)$ \\
Infection & $9(2.7 \%)$ & $6(2.2 \%)$ & $3(4.5 \%)^{*}$ & $12(0.83 \%)$ \\
Sepsis & $18(5.4 \%)$ & $6(2.2 \%)$ & $12(18 \%)^{*}$ & $33(2.3 \%)$ \\
Death & $5(1.5 \%)$ & $4(1.5 \%)$ & $1(1.5 \%)$ & $15(1.0 \%)$ \\
\hline
\end{tabular}

*Denotes statistical significance versus patients treated within $36 \mathrm{~h}, p<0.01$ in all cases 
Table 5 Complications in patients treated surgically for spine fractures versus patients treated for fractures of the pelvis, acetabulum, and/or femur

\begin{tabular}{lll}
\hline & Thoracolumbar spine fracture & Other fractures \\
\hline Fixation $\leq 36 \mathrm{~h}(n=269)$ & 52 patients & 217 patients \\
Any complication & $13(25 \%)^{*}$ & $31(14 \%)$ \\
Pulmonary complication & $11(21 \%)^{*}$ & $18(8.3 \%)$ \\
Fixation $>36 \mathrm{~h}(n=66)$ & 27 patients & 39 patients \\
Any complication & $9(33 \%)^{* *}$ & $13(33 \%)^{* * *}$ \\
Pulmonary complication & $5(19 \%)$ & $2(5.1 \%)$ \\
All patients $(n=335)$ & 79 patients & 256 patients \\
Any complication & $22(28 \%)^{*}$ & $44(16 \%)$ \\
Pulmonary complication & $16(20 \%)^{*}$ & $20(7.8 \%)$ \\
\hline
\end{tabular}

*all $p<0.03$ for patients with spine fractures versus other fractures

${ }^{* *} p=0.04$ for spine patients treated within $36 \mathrm{~h}$ versus later

${ }^{* * *} p=0.002$ for patients without spine fractures treated within $36 \mathrm{~h}$ versus later

isolated injury. Soon, early total care became common, including the fixation of all fractures in the initial surgical setting.

Occasional development of severe complications led some to question early total care for all patients [31-33]. Pape, et al. suggested femoral nailing in patients with lung contusion may cause ARDS. Although early nailing was beneficial in patients without chest injury, $33 \%$ with severe chest injury developed ARDS after early nailing [34]. Fractures cause pain and hemorrhage, and fixation of fractures relieves some of this. However, bleeding and inflammation related to the surgery itself must be considered. This "second hit" in an inadequately resuscitated patient can be associated with deleterious systemic inflammation and organ dysfunction [1,30,33].

Damage control orthopedics (DCO) minimizes surgical duration and bleeding and is an alternative in critically ill patients $[10,11,13,16,19,32]$. Guidelines to stratify patients into stable, borderline, unstable, and in extremis groups based on types of injury to all systems and physiological and laboratory parameters have been proposed, but not validated, suggesting DCO for unstable and in extremis categories [32]. Appropriate patient selection for DCO would be helpful in minimizing the additional costs associated with a second procedure. Furthermore, some femur fractures are not manageable with external fixation. This issue also applies to many pelvic ring, spine, and acetabulum fractures. This group of injuries constitutes a similar set of clinical issues for a trauma patient, in that all of them generate pain and require bedrest and recumbency until they are stabilized. Although the majority of prior literature regarding early fracture care has focused on femoral shaft fractures, benefits of early stabilization of pelvis $[4,6,19,24,35]$, acetabulum $[14,17]$, and spinal fractures have also been described [8, 36-41]. We proposed that one algorithm for patient risk assessment could be developed to address these common trauma scenarios, going beyond treating only the femur [26].

Utility of any treatment algorithm is based on several crucial features. An algorithm must be simple to remember and easy to apply. It should be associated with consistent results or outcomes independent of the individuals involved in the decision-making. Ideally, it would be applicable to a wide group of patients without a change in risk. The EAC protocol was developed by a multi-disciplinary group of physicians, keeping these features in mind. The laboratory parameters are routinely obtained, rapidly available, and low in cost. Serial measurements of venous lactate are more specific than $\mathrm{BE}$ or $\mathrm{pH}$ [42-44], but each of these measurements shows response to resuscitation [45-48]. Over the initial 30 months, protocol violations occurred in only three patients $(0.9 \%)$ for surgery without meeting resuscitative criteria and in 66 resuscitated patients $(20 \%)$ for delayed surgery.

Eighty percent of EAC patients were treated within $36 \mathrm{~h}$, as recommended. Femur fractures were most likely

Table 6 Hospital length of stay (LOS), ICU stay, and ventilation times for EAC patients treated within 36 h of injury versus later. Data are presented as mean and standard deviation

\begin{tabular}{|c|c|c|c|c|}
\hline & All EAC patients $(n=335)$ & $\mathrm{EAC} \leq 36 \mathrm{~h}(n=269)$ & $\mathrm{EAC}>36 \mathrm{~h}(n=66)$ & $p$ value \\
\hline ICU LOS (days) & $6.01 \pm 7.8$ & $4.35 \pm 7.0$ & $11.6 \pm 8.6$ & $<0.0001$ \\
\hline Total LOS (days) & $10.8 \pm 8.1$ & $9.52 \pm 7.8$ & $17.3 \pm 9.1$ & $<0.0001$ \\
\hline Ventilation time (days) & $3.75 \pm 6.6$ & $2.57 \pm 5.7$ & $7.6 \pm 7.0$ & $<0.0001$ \\
\hline
\end{tabular}


to be treated within the recommended time, and spine fractures were most likely to be managed on a delayed basis, consistent with historical practice patterns. Surgeon choice and other nonmedical and operational issues accounted for $89 \%$ of the patients who were delayed. Most often, this was due to preferences to do these cases during regular weekday block time. Patients treated on a delayed basis had higher frequency of both severe head injury and severe abdominal injury; thus, although they met the resuscitative criteria according to laboratory parameters, surgeons may have been more inclined to treat them on a delayed basis. Our analysis attempted to account for these differences through multivariate regression. It is possible that other patient factors, including coagulopathy may have influenced surgeon decision to delay definitive care. Over the 20 months of the study, fewer nonmedical delays occurred. Currently, less than $10 \%$ of our resuscitated patients undergo fixation after $36 \mathrm{~h}$.

The EAC protocol was developed with a system goal to optimize care of multiply-injured patients. The algorithm was based on a predicted reduction of pulmonary and other complications. Prior modeling showed fewer complications when resuscitated patients were treated within $24 \mathrm{~h}$ and to a lesser extent within $48 \mathrm{~h}$ [26]. Thus, $36 \mathrm{~h}$ was chosen as a realistic time frame for adequate resuscitation and for surgeons and operating rooms to be available during daytime hours. We anticipated most patients from other facilities would also arrive within several hours and could be optimized for surgery within the desired timeframe. To date, all patients have met the recommended parameters within $36 \mathrm{~h}$.

When comparing EAC patients who underwent definitive fixation within $36 \mathrm{~h}$ versus the historical group, fewer complications were noted with EAC. Enhancements in critical care and ventilation strategies may have contributed, as the historical group was treated between 2000 and 2007. Our study was underpowered to detect a difference in the rates of pulmonary complications between the EAC and historical groups. With a $20 \%$ rate of nonadherence to the protocol, we would have needed to study 419 patients.

We also hypothesized that EAC patients treated definitively within $36 \mathrm{~h}$ of injury would have fewer complications versus on a delayed basis. Complications were over twice as frequent in patients with delay; sepsis and ARF were significantly more common, but our study is likely underpowered to detect differences in survival. Significantly higher rates of sepsis have been previously reported with delayed fixation. This is believed to be secondary to increased gut permeability to bacterial translocation. Mechanical ventilation, which was significantly prolonged with delayed fixation, has also been shown to increase the tendency for bacterial translocation and infection. Length of stay was also shorter when definitive fixation was undertaken within $36 \mathrm{~h}$, consistent with prior literature which has shown fewer complications and shorter LOS with early surgery for fractures of the femur $[2,3,7,10,12,15,22,49,50]$, pelvis $[4,6,17,24]$, acetabulum [14, 17], or spine [8, 36-41, 51].

O'Toole et al. reviewed patients treated with early intramedullary femoral nailing with lactate $<2.5 \mathrm{mmol} / \mathrm{L}$ and noted acceptably low rates of ARDS when compared with more critically ill patients managed with DCO $(1.5 \%$ versus 0 , NS) [12]. They concluded that with attention to adequate resuscitation, early primary intramedullary nailing is safe in multiple trauma patients. Our patients also developed ARDS infrequently.

Other authors have shown low complication rates when patients were stratified into borderline, unstable, and in extremis groups to determine indications for DCO [19, 32]. We agree in concept with including physiological or laboratory parameters that reflect adequacy of resuscitation. However, we disagree in relegating patients to unstable or other categories, and delayed definitive fixation, purely because they have a severe type of chest, pelvis, and/or extremity injury. It is the hemorrhage associated with some injuries that is problematic, and this bleeding would be reflected in vital signs and laboratory parameters. Furthermore, presence of severe chest injury is a risk factor for pulmonary complications, independent of timing of fixation. Several prior reports have shown that patients with severe chest injury are more likely to develop pulmonary complications when subjected to delayed fixation $[10,11,17,21,51,52]$. Our study supports this, in minimizing complications when resuscitated patients are managed definitively on an early basis.

The strengths of this study include a large sample. All patients were prospectively assessed and treated with this algorithm. One possible criticism is that the threshold for adequacy of resuscitation is too high, potentially creating an unsafe situation for some. However, this algorithm was based on probability modeling in a very large sample, suggesting its safety [26]. The EAC parameters demonstrated reductions in complications when compared with our historical practice, and our results compare favorably with other published data $[7,9,11,15,24,27,50]$.

Another potential criticism of EAC could be failure to adjust threshold parameters based on type of fracture and anticipated surgical duration and hemorrhage. Probst et al. reviewed 213 patients with pelvic ring fractures and concluded that surgery in excess of $3 \mathrm{~h}$ was more often associated with liver failure, while delayed surgery (days 4 to 7 ) may reduce mortality unless surgical duration is under 1 hour, in which case early surgery 
was beneficial [53]. We agree that longer procedures carry more surgical risk, in terms of infectious and thrombotic complications. However, our data suggest that in an adequately resuscitated patient, earlier definitive surgery appears acceptable with fewer complications than when the same surgery is delayed. We believe that fracture stabilization is part of the resuscitation, as it reduces pain and bleeding. Our algorithm encourages continuous reassessment during procedures to ensure that resuscitation is occurring, in order to account for bleeding generated from the surgery itself.

Several limitations to the current EAC protocol were noted by our team over the course of study. Patients of advanced age or with medical risk factors may require additional cardiac or other diagnostic tests as part of their pre-operative risk assessment and optimization. This occurred in $<1 \%$ of our patients. Occasionally, patients have met the prescribed resuscitation criteria but were critically ill due to severe head injury with elevated intracranial pressure, evolving myocardial ischemia or infarct, or other medical reasons. Such issues required delay in $2 \%$ of our patients.

\section{Conclusions}

In summary, definitive stabilization of fractures of the femur, pelvis, spine, and acetabulum should occur when patients are adequately resuscitated to prevent a deleterious reactive systemic inflammatory response. Our protocol recommends definitive fixation within $36 \mathrm{~h}$ in resuscitated patients as defined by at least one of the following: venous lactate $<4.0 \mathrm{mmol} / \mathrm{L}, \mathrm{BE} \geq-5.5 \mathrm{mmol} / \mathrm{L}$, or $\mathrm{pH} \geq 7.25$. All 335 patients had achieved the desired level of resuscitation within that time. Some were treated on a delayed basis for medical reasons, but surgeon choice and other nonmedical and operational issues accounted for $89 \%$ of delays. Early fracture care resulted in fewer complications and shorter LOS in EAC patients, and EAC patients had fewer complications than our historical group, when controlling for age, timing of fixation, and severity of other injuries, suggesting improvement with a standardized protocol to assess adequacy of resuscitation. EAC recommendations appear safe.

\section{Competing interests}

The authors declare that they have no competing interests.

\begin{abstract}
Authors' contributions
HAV, TAM, JJC, XW, and AJD designed the study. TAM, KGW, and AJD collected the data. XW analyzed the data. HAV, TAM, JJC, PAW, MPS, KGW CES, and AJD critically reviewed the data. AJD developed data tables for presentation. HAV and XW wrote the manuscript. TAM, JJC, PAW, and CES critically reviewed and revised the manuscript. All authors read and approved the final manuscript.
\end{abstract}

\section{Acknowledgements}

The authors gratefully acknowledge all trauma providers in the MetroHealth System for their tireless efforts to deliver outstanding trauma care and to continuously strive for improvement. We acknowledge Alysse J. Boyd for assistance with data collection.

We also thank the adjudication committee members: Megan A. Brady, MD; Sean J. Nagel, MD; David T. Porembka, DO; and Charles J. Yowler, MD for their efforts throughout this study and analysis.

This study was not externally funded.

\section{Author details}

${ }^{1}$ Department of Orthopaedic Surgery, MetroHealth Medical Center affiliated with Case Western Reserve University, 2500 MetroHealth Drive, Cleveland, $\mathrm{OH} 44109$, USA. ${ }^{2}$ Departments of Orthopaedic Surgery and Neurosciences, MetroHealth Medical Center affiliated with Case Western Reserve University, 2500 MetroHealth Drive, Cleveland, OH, USA. ${ }^{3}$ Division of Trauma,

Department of Surgery, MetroHealth Medical Center affiliated with Case Western Reserve University, Cleveland, $\mathrm{OH}$, USA. ${ }^{4}$ Department of Neurosciences, MetroHealth Medical Center affiliated with Case Western Reserve University, Cleveland, OH, USA. ${ }^{5}$ Department of Anesthesiology, MetroHealth Medical Center affiliated with Case Western Reserve University, Cleveland, $\mathrm{OH}$, USA.

Received: 27 May 2015 Accepted: 20 September 2015

Published online: 01 October 2015

\section{References}

1. Balogh ZJ, Reumann MK, Gruen RL, Mayer-Kuckuk P, Schuetz MA, Harris IA, et al. Advances and future directions for management of trauma patients with musculoskeletal injuries. Lancet. 2012;380:1109-19.

2. Bone LB, Johnson KD, Weigelt J, Scheinberg R. Early versus delayed stabilization of femoral fractures. A prospective randomized study. J Bone Joint Surg Am. 1989;71:336-40.

3. Brundage SI, McGhan R, Jurkovich GJ, Mack CD, Maier RV. Timing of femur fracture fixation: effect on outcome in patients with thoracic and head injuries. J Trauma. 2002;52:299-307.

4. Goldstein A, Phillips T, Sclafani SJ, Scalea T, Duncan A, Goldstein J, et al. Early open reduction and internal fixation of disrupted pelvic ring. J Trauma. 1986;26:325-33.

5. Johnson KD, Cadambi A, Seibert GB. Incidence of adult respiratory distress syndrome in patients with multiple musculoskeletal injuries: effect of early operative stabilization of fractures. J Trauma. 1995;25:375-84.

6. Latenser BA, Gentilello LM, Tarver AA, Thalgott JS, Batdorf JW. Improved outcome with early fixation of skeletally unstable pelvic fractures. J Trauma. 1991;31:28-31.

7. Lefaivre KA, Starr AJ, Stahel PF, Elliott AC, Smith WR. Prediction of pulmonary morbidity and mortality in patients with femur fracture. J Trauma. 2010;69:1527-36.

8. McHenry TP, Mirza SK, Wang J, Wade CE, O'Keefe GE, Dailey AT, et al. Risk factors for respiratory failure following operative stabilization of thoracic and lumbar spine fractures. J Bone Joint Surg Am. 2006;88:997-1005.

9. Morshed S, Miclau T, Bembom O, Cohen M, Knudson MM, Colford Jr JM. Delayed internal fixation of femoral shaft fracture reduces mortality among patients with multisystem trauma. J Bone Joint Surg Am. 2009;91:3-13.

10. Nahm N, Como JJ, Wilber JH, Vallier HA. Early appropriate care: definitive stabilization of femoral fractures within 24 hours is safe in most multiply-injured patients. J Trauma. 2011;71:175-85.

11. Nahm NJ, Vallier HA. Timing of definitive treatment of femoral shaft fractures in multiple trauma patients: a systematic review. J Trauma Acute Care Surg. 2012;73:1046-63.

12. O'Toole RV, O'Brien M, Scalea TM, Habashi N, Pollak AN, Turen CH. Resuscitation before stabilization of femoral fractures limits acute respiratory distress syndrome in patients with multiple traumatic injuries despite low use of damage control orthopaedics. J Trauma. 2009;67:1013-21.

13. Pape HC, Hildebrand F, Pertschy S, Zelle B, Garapati R, Grimme K, et al. Changes in the management of femoral fractures in polytrauma patients: from early total care to damage control orthopedic surgery. J Trauma. 2002;53:452-61.

14. Plaisier BR, Meldon SW, Super DM, Malangoni MA. Improved outcome after early fixation of acetabular fractures. Injury. 2000;31:81-4.

15. Rixen D, Grass G, Sauerland S, Lefering R, Raum MR, Yucel N, et al. Evaluation of criteria for temporary external fixation in risk-adapted damage control orthopedic surgery of femur shaft fractures in multiple trauma 
patients: "evidence-based medicine" versus "reality" in the trauma registry of the German Trauma Society. J Trauma. 2005;59:1375-94.

16. Scalea TM, Boswell SA, Scott JD, Mitchell KA, Kramer ME, Pollak AN. External fixation as a bridge to intramedullary nailing for patients with multiple injuries and with femur fractures: damage control orthopedics. J Trauma. 2000;48:613-23.

17. Vallier HA, Cureton BA, Eckstein C, Oldenburg FP, Wilber JH. Early definitive stabilization of unstable pelvis and acetabulum fractures reduces morbidity. J Trauma. 2010;69:677-84.

18. Vallier HA, Super DM, Moore TA, Wilber JH. Do patients with multiple system injury benefit from early fixation of unstable axial fractures? The effects of timing of surgery on initial hospital course. J Orthop Trauma. 2013;27:405-12.

19. Pape HC, Rixen D, Morley J, Husebye EE, Mueller M, Dumont C, et al. Impact of the method of initial stabilization for femoral shaft fractures in patients with multiple injuries at risk for complications (borderline patients). Ann Surg. 2007;246:491-9.

20. Tuttle MS, Smith WR, Williams AE, Agudelo JF, Hartshorn CH, Moore EE, et al. Safety and efficacy of damage control external fixation versus early definitive stabilization for femoral shaft fractures in the multiple-injured patient. J Trauma. 2009:67:602-5.

21. Seibel R, LaDuca J, Hassett JM, Babikian G, Mills B, Border DO, et al. Blunt multiple trauma (ISS 36), femur traction, and the pulmonary failure-septic state. Ann Surg. 1985;202:283-95.

22. Starr AJ, Hunt $J$, Chason DP, Reinert CM, Walker J. Treatment of femur fracture with associated head injury. J Orthop Trauma. 1998;12:38-45.

23. White TO, Jenkins PJ, Smith RD, Cartlidge CW, Robinson CM. The epidemiology of posttraumatic adult respiratory distress syndrome. J Bone Joint Surg Am. 2004;86:2366-76.

24. Enninghorst N, Toth L, King KL, McDougall D, Mackenzie S, Balogh ZJ. Acute definitive internal fixation of pelvic ring fractures in polytrauma patients: a feasible option. J Trauma. 2010;68:935-41.

25. Vallier HA, Cureton BA, Patterson BM. Factors affecting revenue from management of pelvis and acetabulum fractures. J Orthop Trauma. 2013;27:267-74.

26. Vallier HA, Wang $\mathrm{X}$, Moore TA, Wilber $\mathrm{JH}$, Como JJ. Timing of orthopaedic surgery in multiply-injured patients: development of a protocol for early appropriate care. J Orthop Trauma. 2013;27:543-51.

27. Dellinger RP, Levy MM, Carlet JM, Bion J, Parker MM, Jaeschke R, et al. International Surviving Sepsis Campaign Guidelines Committee. Surviving sepsis campaign: international guidelines for management of severe sepsis and septic shock: 2008. Crit Care Med. 2008;36:296-327.

28. Bellomo R, Ronco C, Kellum JA, Mehta RL, Palevsky P, Acute Dialysis Quality Initiative workgroup. Acute renal failure-definition, outcome measures, animal models, fluid therapy and information technology needs: the second international consensus conference of the acute dialysis quality initiative (ADQI) group. Crit Care. 2004;8:R204-12.

29. Durham RM, Moran JJ, Mazuski JE, Shapiro MJ, Baue AE, Flint LM. Multiple organ failure in trauma patients. J Trauma. 2003;55:608-16.

30. American Thoracic Society. Documents approved by ATS Board of Directors, Dec 2004. Guidelines for the management of adults with hospital-acquired, ventilator-associated, and healthcare-associated pneumonia. Am J Respir Crit Care Med. 2005;171:388-416.

31. Harwood PJ, Giannoudis PV, van Griensven M, Krettek C, Pape HC. Alterations in the systemic inflammatory response after early total care and damage control procedures for femoral shaft fracture in severely injures patients. J Trauma. 2005:58:446-52.

32. Pape HC. Effects of changing strategies of fracture fixation on immunologic changes and systemic complications after multiple trauma. J Orthop Res. 2008:26:1478-84

33. Pape HC, van Griensven MV, Hildebrand FF, Tzioupis $C T$, Sommer KL, Krettek CC, et al. Systemic inflammatory response after extremity or truncal fracture operations. J Trauma. 2008;65:1379-84.

34. Pape HC, Regel G, Dwenger A, Sturm JA, Tscherne H. Influence of thoracic trauma and primary femoral nailing on the incidence of ARDS in multiple trauma patients. Injury. 1993;24 Suppl 3:S82-103.

35. Barei DP, Shafer BL, Beingessner DM, Gardner MJ, Nork SE, Routt ML. The impact of open reduction internal fixation on acute pain management in unstable pelvic ring injuries. J Orthop Trauma. 2010;24:263-70.

36. Cengiz SL, Kalkan E, Bayir A, llik K, Basefer A. Timing of thoracolumbar spine stabilization in trauma patients; impact on neurological outcome and clinical course. A real prospective (rct) randomized controlled study. Arch Orthop Trauma Surg. 2008;128:959-66.

37. Dimar JR, Carreon LY, Riina J, Schwartz DG, Harris MB. Early versus late stabilization of the spine in the polytrauma patient. Spine. 2010;35(21 Suppl):S187-92.

38. Frangen TM, Ruppert S, Muhr G, Schinkel C. Respiratory failure in thoracic spine injuries. Does the timing of dorsal stabilization have any effect on the clinical course in multiply injured patients? Orthopaedics 2007;36:365-71.

39. Kerwin AJ, Griffen MM, Tepas JJ, Schinco MA, Devin T, Frykberg ER. Best practice determination of timing of spinal fracture fixation as defined by analysis of the National Trauma Data Bank. J Trauma. 2008;65:824-31.

40. Schinkel C, Frangen TM, Kmetic A, Andress HJ, Muhr G, German Trauma Registry. Timing of thoracic spine stabilization in trauma patients: impact on clinical course and outcome. J Trauma. 2006;61:156-60.

41. Stahel PF, Flierl MA, Moore EE, Smith WR, Beauchamp KM, Dwyer A. Advocating "spine damage control" as a safe and effective treatment modality for unstable thoracolumbar fractures in polytrauma patients: a hypothesis. J Trauma Mgmt Outcomes. 2009;3:6-9.

42. Guyette F, Suffoletto B, Castillo J, Quintero JL, Callaway C, Puyana JC. Prehospital serum lactate as a predictor of outcomes in trauma patients: a retrospective observational study. J Trauma. 2011;70:782-6.

43. Husain FA, Martin MJ, Mullenix PS, Steele SR, Elliott DC. Serum lactate and base deficit as predictors of mortality and morbidity. Am J Surg. 2003;185:485-91.

44. Paladineo L, Sinert R, Wallace D, Anderson T, Yadav K, Zehtabchi S. The utility of base deficit and arterial lactate in differentiating major from minor injury in trauma patients with normal vital signs. Resuscitation. 2008;77:363-8.

45. Claridge JA, Crabtree TD, Pelletier SJ, Butler K, Sawyer RG, Young JS. Persistent occult hypoperfusion is associated with a significant increase in infection rate and mortality in major trauma patients. J Trauma. 2000;48:8-14.

46. Crowl AC, Young JS, Kahler DM, Claridge JA, Chrzanowski DS, Pomphrey M Occult hypoperfusion is associated with increased morbidity in patients undergoing early femur fracture fixation. J Trauma. 2000;48:260-7.

47. Davis JW, Parks SN, Kaups KL, Gladen HE, O'Donnell-Nicol S. Admission base deficit predicts transfusion requirements and risk of complications. J Trauma. 1996:41:769-74.

48. Eberhard LW, Morabito DJ, Matthay MA, Mackersie RC, Campbell AR, Marks JD, et al. Initial severity of metabolic acidosis predicts the development of acute lung injury in severely traumatized patients. Crit Care Med. 2000;28:125-31.

49. Scalea TM, Scott JD, Brumback RJ, Burgess AR, Mitchell KA, Kufera JA, et al. Early fracture fixation may be "just fine" after head injury: no difference in central nervous system outcomes. J Trauma. 1999:46:839-46.

50. Nicholas B, Toth L, van Wessem K, Evans J, Enninghorst N, Balogh ZJ. Borderline femur fracture patients: early total care or damage control orthopaedics? ANZ J Surg. 2011;81:148-53.

51. Bellabarba C, Fisher C, Chapman JR, Dettori JR, Norvell DC. Does early fracture fixation of thoracolumbar spine fractures decrease morbidity or mortality? Spine. 2010;35(9 Suppl):S138-45.

52. Bosse MJ, MacKenzie EJ, Riemer BL, Brumback RJ, McCarthy ML, Burgess AR, et al. Adult respiratory distress syndrome, pneumonia, and mortality following thoracic injury and a femoral fracture treated either with intramedullary nailing with reaming or with a plate. A comparative study. $J$ Bone Joint Surg Am. 1997;79:799-809.

53. Probst C, Probst T, Gaensslen A, Krettek C, Pape HC, Polytrauma Study Group of the German Trauma Society. Timing and duration of the initial pelvic stabilization after multiple trauma in patients from the German trauma registry: Is there an influence on outcome? J Trauma. 2007;62:370-7. 